Research Article

\title{
Reliability Optimization of Structural Deformation with Improved Support Vector Regression Model
}

\author{
Zheng-Zheng Zhu, ${ }^{1}$ Yun-Wen Feng $\mathbb{D}^{1},{ }^{1}$ Cheng Lu $\mathbb{D}^{1},{ }^{1}$ and Cheng-Wei Fei $\mathbb{D}^{2}$ \\ ${ }^{1}$ School of Aeronautics, Northwestern Polytechnical University, Xi'an 710072, Shaanxi, China \\ ${ }^{2}$ Department of Aeronautics and Astronautics, Fudan University, Shanghai 200433, China \\ Correspondence should be addressed to Cheng Lu; lucheng2013@163.com
}

Received 30 January 2020; Revised 17 February 2020; Accepted 19 February 2020; Published 18 March 2020

Guest Editor: Shun-Peng Zhu

Copyright (c) 2020 Zheng-Zheng Zhu et al. This is an open access article distributed under the Creative Commons Attribution License, which permits unrestricted use, distribution, and reproduction in any medium, provided the original work is properly cited.

\begin{abstract}
Deformation is one important failure mode of turbine blades. The quality of a model seriously influences the reliability optimization of turbine blades in turbo machines. To improve the reliability optimization of turbine blades, this paper proposes a novel machine learning-based reliability optimization approach, named improved support vector regression (SR) model (ISRM) method, by fusing artificial bee colony (ABC), traditional SR model, and multipopulation genetic algorithm (MPGA). In this proposed method, the ABC algorithm was applied to find the optimal parameters in the SR model to establish accurate ISRM, following the thought of the surrogate model method and the randomness of constraints. Then the reliability optimization model and procedure with the ISRM method were resolved by the MPGA. Regarding many design parameters (i.e., rotor speed, temperature, and aerodynamic pressure), design objective (i.e., blade deformation), and the randomness constraints of reliability degree and boundary conditions, we performed the reliability optimization of a turbine blade deformation. From the optimization results, we find that the turbine blade deformation is reduced by $0.09329 \mathrm{~mm}$, and the ISRM learning method can improve the reliability optimization design of complex structures with the emphasis on modeling precision and optimization efficiency. The works of this paper provide a machine learning-based reliability optimization approach for the reliability optimization of complex structures and enrich and develop mechanical reliability theory and methods.
\end{abstract}

\section{Introduction}

The reliability optimization design of mechanical structures is to find the optimal design parameters by establishing the reliability optimization model, subject to the related parameters (i.e., material properties and complex loads), reliability index, and the technical and economic requirements [1]. The reliability optimization of complex structures generally involves failure mode, such as stress, strain, and deformation, and has the properties of nonlinearity and randomness [2]. Herein, structural deformation is a typical failure mode for turbine blade, and the failure mode of turbine blade is induced by the randomness of workloads and material parameters (such as temperature, rotor speed, aerodynamic pressure, density, elastic modulus, and thermal conductivity) and constraints as well [3]. Thus, structural reliability optimization has large-scale computational load and complexity and is harshly performed with an acceptable efficiency and accuracy by finite element (FE) simulation and stochastic optimization methods. To let the optimization design results cater for the engineering requirements, it is necessary to explore an effective approach for the reliability optimization of mechanical structure.

With the requirement of design analysis for advanced mechanical systems, many reliability optimization methods have emerged in engineering practice [4-9]. Surrogate model method (also called response surface method) is a promising way to ensure efficiency and accuracy in structural reliability optimization, instead of the direct simulation method with the FE model [4]. Fei et al. developed extremum surrogate model methods with quadratic polynomials and support vector regression, for 
dynamic reliability optimization design of turbine blade-tip clearance [10] and its components [11, 12]. Song et al. [13] studied multisurrogate models with a backpropagationartificial neural network (BP-ANN), for the multiobjective reliability optimization of turbine blisk, considering fluidthermal-structure interaction. Song et al. [14] discussed the constraint-feasible moving-least-squares method for the reliability optimization of floating production storage and offloading river support structures, regarding running status, damage, and loadings. Youn and Choi [15] gave an improved RSM with the moving-least-squares method and the hybrid mean-value method, to investigate the reliability optimization of vehicle side and improve its crashworthiness. Meng et al. [16] introduced a saddle point approximation reliability analysis method, combined with collaborative optimization method, to solve turbine blade unsafe design problem. Although the above works investigated structural reliability optimization using different surrogate modeling strategies, the analytical results cannot reach the engineering requirements owing to the limitation of optimization models. Hence, it is unworkable for these methods to handle the reliability optimization problems of complex structures with acceptable accuracy and efficiency.

At present, the machine learning method has got a rapid development in data-derived modeling and image processing [17-19]. The heuristic method prompts the author to expand this idea to engineering optimization. As one of the typical machine learning methods developed from statistical learning theory, support vector machine (SVM) of regression (denoted by SR) has an excellent learning ability of small samples and highly efficient simulation ability and has been validated in the reliability optimization design [20-25]. Recently, Brabanter et al. [20] developed the least-squares SVM (LSSVM) by transforming the optimization problem of SR into a quadratic programming problem with a single equation constraint to accelerate support vector regression solution. However, the SR is susceptible to noise and outliers when training samples contain multiple input parameters. In addition, it is unable to find the optimal values of the SR model for multivariable and high-nonlinearity problems. Therefore, we need to search effective way to derive structural reliability optimization designs.

The goal of this paper is to open a new research direction, that is, machine learning-based reliability optimization, for mechanical structures, with respect to the randomness of design parameters and constraints. Namely, an improved model (ISRM) method is developed. In this method, an artificial bee colony (ABC) algorithm [26] is used to find the optimal parameters of SR models. The reliability optimization of a turbine blade deformation is completed by the proposed ISRM method with a multipopulation genetic algorithm (MPGA) [27]. Besides, the proposed approach is validated by the comparison of methods.

The remains of this paper are organized as follows. In Section 2, the ISRM method is investigated, comprising the thought of the ISRM method for turbine blade reliability optimization, the mathematical modeling of the ISRM method, and the reliability optimization model. In Section 3, the reliability optimization of turbine blade deformation is finished with the proposed ISRM method. Section 4 summarizes the main conclusions of this study.

\section{Methods and Models}

Support vector machine (SVM) is one of the typical machine learning methods. The SVM was first proposed in data mining by regression and classification which are called SVM of regression (SR) and SVM of classification (SC) $[28,29]$. The basis of an SVM model is statistical learning algorithm so that the SVM is suitable for small samples of structural design analysis, which are gained from a few FE simulations. The SR model is good at the solution of high nonlinear problems between input variables and output response, by introducing a maximum classification margin subject to inequality constraints [30]. Hence, the SR can improve the computational efficiency and accuracy of structural reliability optimization [9]. As for large-scale parameters and high-nonlinearity problems, the traditional SR is easy to fall into local optimization in the process of searching hyperparameter, which affects the precision of structural reliability optimization. In most of the structural probabilistic designs, the number of influential parameters and constraint conditions largely impacts the precision of design. Therefore, this paper considers the effect of design parameters and constraint conditions and develops a machine learning-based reliability optimization approach, that is, improved SR model (ISRM) method, based on the artificial bee colony ( $\mathrm{ABC}$ ) algorithm, traditional SR model, and multipopulation genetic algorithm (MPGA), to accomplish the reliability optimization of turbine blade deformation.

2.1. Improved Support Vector Regression Model. When $\mathbf{X}$ and $y(\mathbf{X})$ indicate input sample set of a component in structure system and the corresponding output response, respectively, the response curve was constructed by the sample set $\{y(\mathbf{X})\}$. The relationship between $\mathbf{X}$ and $y$ is written as

$$
y=f(\mathrm{X})=\{y(\mathrm{X})\} .
$$

The SR method with failure mode is modeled as

$$
y=f(\mathbf{x})=\sum_{i=1}^{l} \mathbf{a}_{i} \mathbf{K}\left(\mathbf{x}, \mathbf{x}_{i}\right)+b,
$$

where $l$ is the number of support vectors in the sample set; $y$, $\mathbf{a}_{i}$, and $b$ indicate the output response, weight vector, and a bias term; and $\mathbf{K}\left(\mathbf{x}, \mathbf{x}_{i}\right)$ is the kernel function of the failure mode. Kernel function includes linear kernel function, polynomials kernel function, radial basis function (RBF) kernel function, sigmoid kernel function, and Gauss kernel function. In this study, the Gauss function is selected as the kernel function of SR model. Thus, the kernel function of the SR model for the failure mode is expressed as

$$
\mathbf{K}\left(\mathbf{x}, \mathbf{x}_{i}\right)=\exp \left(-\frac{\left\|x_{i}-x^{\prime}\right\|^{2}}{2(\sigma)^{2}}\right),
$$


in which $x_{i}$ is $i$ th sample in SR model; $x^{\prime}$ is the center point of sample set; $\sigma$ is the width of kernel function $\mathbf{K}\left(\mathbf{x}, \mathbf{x}_{i}\right)$.

Assume that $\boldsymbol{\theta}=\left[\mathbf{a}_{i}, \mathbf{b}_{i}, \sigma\right]$ denotes the parameters of SR model. Because $\boldsymbol{\theta}$ seriously influences the precision of SR model, it is reasonable to find the optimal parameters $\boldsymbol{\theta}$ to guarantee the precision of SR modeling in equation (2). The traditional search method for the parameters $\boldsymbol{\theta}$ possesses some blindness because the effect of search seriously depends on the experience. Thus, it is urgent to develop an efficient algorithm to find the parameters $\boldsymbol{\theta}$ in SR modeling.

To effectively determine the optimal values of the parameters $\boldsymbol{\theta}$ in the ISRM, the $\mathrm{ABC}$ algorithm is applied to derive the solution of objective function in this paper. The $\mathrm{ABC}$ algorithm is a global optimization algorithm based on group intelligence proposed to by Karaboga in 2005, which is used to accomplish the solution of nonlinear problems [26]. Its feature is that this algorithm obtains the global optimal value of population via comparison of the merits and demerits of the problem and the local optimization behavior of each individual artificial bee, and this method has a faster convergence speed [31-33]. The basic principle of the ABC algorithm is shown as below. The honey collecting system is composed of food source, employed bees, and unemployed bees, and unemployed bees include on-looker bees and scout bees. Herein, the employed bees are associated with the specific food source and tell the information of food source to the companion in a certain probability form; and the unemployed bees are applied to search food source. In the process of finding the parameters $\boldsymbol{\theta}$, a part of the colony is treated as scout bees to search for the food source. If the quantity of a food source exceeding a certain threshold is found, the scout bees become employed bees and start to collect food. After collecting food, the employed bees transmit the information for food source to the on-looker bees, and the on-looker bees can accurately evaluate the quality of food source. The optimal values of the parameters $\boldsymbol{\theta}$ in the ISRM are determined via multiple iterations.

\subsection{Reliability Optimization Model with Improved Support} Vector Regression Model. In this reliability optimization, the sensitivity analysis of random variables on output response is performed to select highly sensitive input random variables as design variables. The output responses, that is, overall stress and deformation of structure, are regarded as design objectives, and the reliability $R$ of the turbine blade and performance constraints allowable value $\left[\sigma_{0}\right]$ are taken as constraints. Therefore, the reliability optimization model of mechanical structures with the ISRM method is expressed as

$$
\text { Find } \mathbf{x}=\left(x_{1}, x_{2}, \ldots, x_{n}\right)^{T},
$$

$\min f(\mathbf{x})$,

$$
\text { s.t. } \quad\left\{\begin{array}{l}
R \geq\left[R_{0}\right], \\
f(\mathbf{x}) \leq\left[\sigma_{0}\right], \\
\underline{x}_{i} \leq x_{i} \leq \bar{x}_{i,} \\
\underline{x}_{i}=x_{i}^{l}+\lambda^{*}\left(x_{i}^{u}-x_{i}^{l}\right), \\
\bar{x}_{i}=x_{i}^{u}-\lambda^{*}\left(x_{i}^{u}-x_{i}^{l}\right),
\end{array}\right.
$$

where $R$ and $\left[R_{0}\right]$ stand for the reliability degree and allowable reliability, respectively; $\underline{x}_{i}, \bar{x}_{i}$ denote upper and lower boundary of $i$ th fuzzy variables. The upper and lower bounds of the transitional interval are determined by introducing amplification coefficient; that is, $\underline{x}^{n}=\underline{x}$, $\underline{\underline{x}}^{l}=\beta \times \underline{x} ; \bar{x}^{u}=\bar{\beta} \times \bar{x}, \bar{x}^{l}=\bar{x} ;\{\boldsymbol{\sigma}\}=\left[\sigma_{x}, \sigma_{y}, \sigma_{z}, \tau_{x y}, \tau_{y z}, \tau_{z x}\right] ;$ $\bar{\beta}=\overline{1.1} \sim 1.3 ; \lambda^{*}$ expresses the optimal level cut set.

The MPGA [27] is adopted to derive the solution of objective function equation (4). Comparing with the GA, the MPGA holds more flexible and adaptive design space exploration, which has the potential to avoid the effect of plateau-like function [34]. Besides, the MPGA breaks the limitation of a single population evolution of GA and uses many populations with different control parameters for optimization iterations [29, 30]. Besides, the MPGA derived from GA inherits natural selection and genetic properties, and the optimal solution of the objective function can be gained via enough iterations with selection, crossover, and mutation. The basic principle of the MPGA is summarized below. Firstly, after generating $N$ initial populations (i.e., blade density, rotor speed, temperature, aerodynamic pressure, and gravity) with binary encoding, we gain the $N$ new populations by selection operator, crossover operator, and mutation. Then, the optimal individuals of each excellent population are selected via artificial selection operator and then are regarded as structure elite population to search for the optimal value of objective function. In this case, the MPGA is essentially a combination of multiple GAs in line with a specific relationship. However, in the MPGA, the control parameters, such as crossover probability and mutation probability, are applied to complete the collaborative evaluation of multiple populations. Therefore, the MPGA has both global and local search abilities by introducing immigrant operator to exchange information among populations and avoid the destruction and loss of optimal individual information. In this process, the elite population does not participate as selection, crossover, and mutation operators. The minimum reserved generation is usually regarded as the terminal condition of optimization iterations. Through the above steps, the reliability optimization of mechanical structures can be finished based on the ISRM method and MPGA.

\subsection{Flowchart of Reliability Optimization with Improved} Support Vector Regression Model. In line with the ISRM method, the procedure of turbine blade deformation reliability optimization is shown as follows. Firstly, the FE model of turbine blades is built and blade density, rotor speed, temperature, aerodynamic pressure, and gravity are selected as input variables, and the deformation of turbine blades is considered as failure mode, subject to the randomness of rotor speed, gas temperature, and boundary conditions. Secondly, in respect of FE model, thermalstructural interaction, and the means of random variables, the static deterministic analysis of turbine blade is conducted to find the maximum points of blade deformation as the 
object of turbine blade optimization. Thirdly, regarding the randomness of design parameters, handful samples of random variables are extracted by Latin hypercube sampling (LHS) technique [35], and by FE simulations with the extracted samples, the output response of stress and deformation are calculated as output samples. Next, normalizing input samples and output samples as the training samples is to find the optimal parameters of the SR model by using an artificial bee colony (ABC) algorithm [29] and then to build ISRM for the deformation of turbine blades. Then, by acquiring enough samples by the linkage LHS technique [35], the probabilistic simulations of mechanical structure are completed based on the developed ISRM method. Following that, the reliability optimization model with the developed ISRM method is established by adopting the MPGA to seek for the optimal parameters in the reliability optimization. Lastly, the reliability optimization of turbine blade deformation with the ISRM method is implemented to find the optimal parameters subject to design objectives and constraints.

The above analysis flowchart is summarized in Figure 1.

\section{Reliability Optimization of Turbine Blade Deformation}

In this section, the developed ISRM method will be adopted to investigate the reliability optimization of an aeroengine turbine blade deformation. Blade material is GH4133B alloy [36]. Based on the random variable selection method [9], blade density, rotor rotational speed, gas temperature, gas pressure, and gravity acceleration are termed input random variables (parameters). The distribution features of the parameters are listed in 1, in which all the variables follow normal distributions of mutual independence.

The FE model of the turbine blade is established, which include 98569 elements and 163312 nodes as displayed in Figure 2. In respect of the mean values of parameters in Table 1, the steady deterministic analyses of blade radial deformation were performed. The distributions of turbine blade deformation are drawn in Figure 3 as the analysis was stable.

In Figure 3, it is illustrated that the maximum deformation is $1.021 \mathrm{~mm}$ at the blade top. Therefore, the deformation is considered to investigate the blade reliability optimization with deformation failures in coming part.

\subsection{Turbine Blade Optimization with Improved Support Vector Regression Model}

3.1.1. Improved Support Vector Regression Modeling. By the LHS technique [35], the input variables in Table 1 were collected at the critical point when turbine blade deformation reached the maximum. Namely, the key points were the maximum deformation obtained based on the above static deterministic analyses, and the maximum deformation was acquired based on the static analyses with the extracted samples. The normalized samples were termed the training samples for SR modeling. To improve SR modeling accuracy,

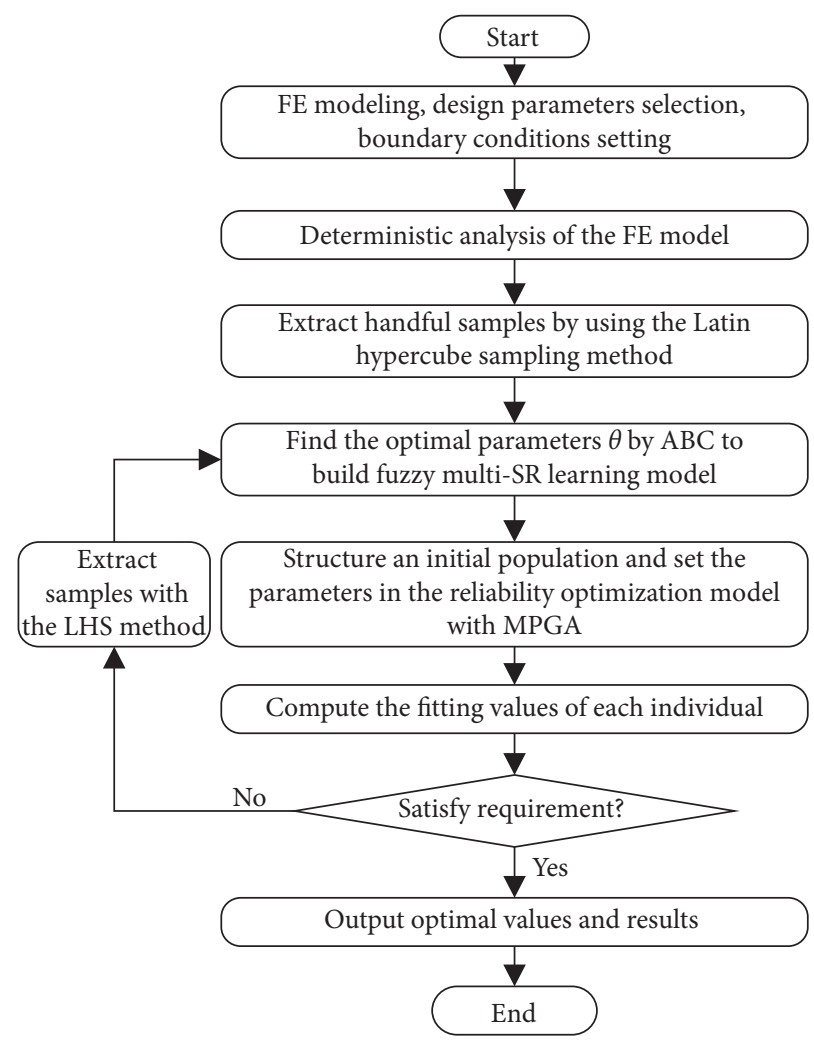

FIGURE 1: Reliability optimization procedure with the ISRM method.

TABle 1: Distribution features of input parameters.

\begin{tabular}{lccc}
\hline Parameters & Mean & St. dev. & Distribution \\
\hline Rotational speed $\omega, \mathrm{rad} / \mathrm{s}$ & 1160 & 58 & Normal \\
Gas temperature $T, \mathrm{~K}$ & 1200 & 60 & Normal \\
Blade density $\rho, \mathrm{kg} / \mathrm{m}^{3}$ & 8200 & 246 & Normal \\
Gas pressure $P, \mathrm{MPa}$ & 0.5 & 0.025 & Normal \\
Acceleration of gravity $g, \mathrm{~m} / \mathrm{s}^{2}$ & 9.81 & 0.2943 & Normal \\
\hline
\end{tabular}

the $A B C$ technique [29] was employed to optimize the parameters $\boldsymbol{\theta}=(c, \sigma, \varepsilon)$ in SR modeling. The coefficients of ISRM for turbine blade deformation are shown in equation (5). In the next section, we will employ the established ISRM to complete the reliability optimization of turbine blade deformation.

$$
\left\{\begin{array}{l}
a=\left(\begin{array}{ccc}
13.4221 & -2.5412 & 16.4211 \\
3.2121 & 3.5723 & -10.5421 \\
-8.4422 & 9.4542 & 17.5224
\end{array}\right) \\
{[c, \sigma, \varepsilon]=[125.4555,107.5244,2.7789]} \\
b=0.7478
\end{array}\right.
$$

3.1.2. Turbine Blade Deformation Optimization. For accomplishing the reliability optimization of turbine blade deformation, the maximum deformation $\delta_{\max }$ of the turbine blade is minimized by adopting the structured ISRM. The input variables $(\omega, T)$ are respected as design 


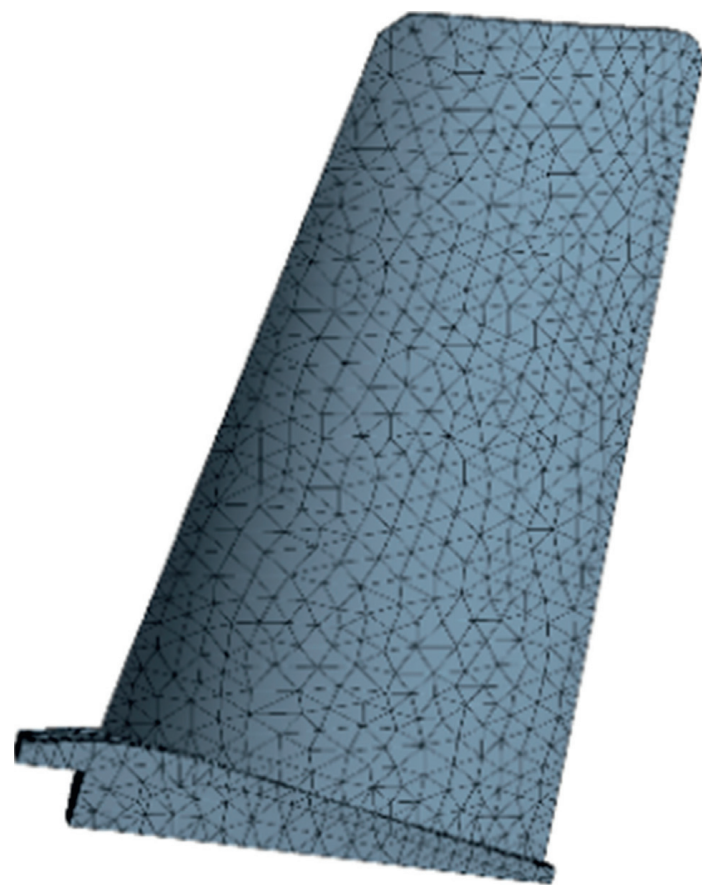

Figure 2: FE model of turbine blade.

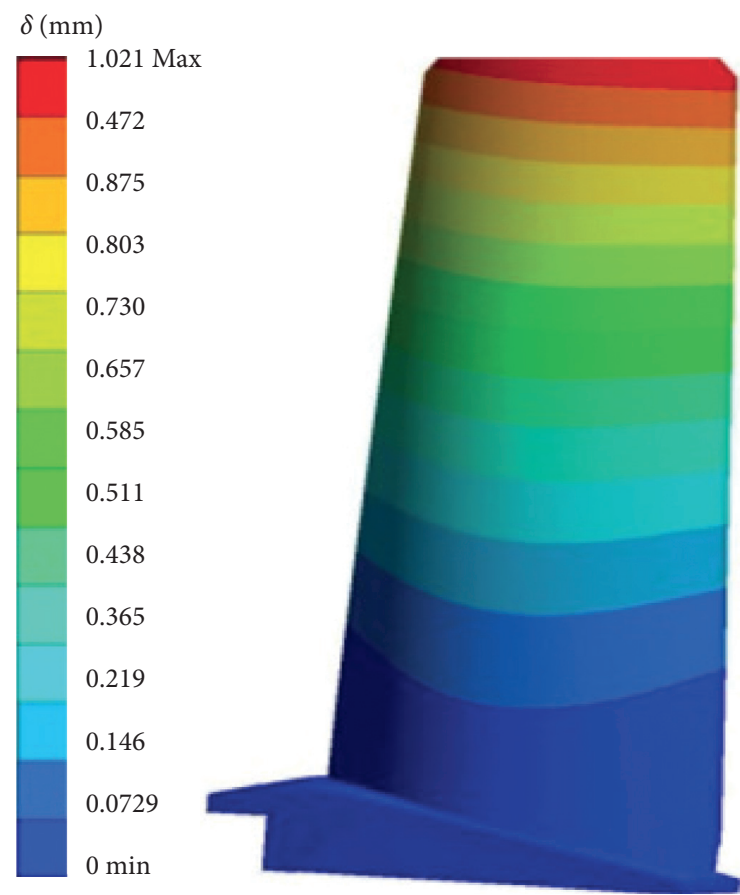

Figure 3: Blade deformation distribution.

variables, and turbine blade deformation $\delta$ is regarded as design objective function, and the reliability index and boundary loads are considered as constraint conditions. The reliability optimization model of turbine blade deformation is shown in equation (6). Herein, the upper and lower limits of constraints are revealed in Table 2, where the values of upper and lower limits are determined by the 3 sigma levels $[3,5]$.
TABle 2: Constraints of design parameters.

\begin{tabular}{ccccc}
\hline \multicolumn{2}{c}{ Upper and lower limit } & {$[\delta], \mathrm{mm}$} & $\omega, \mathrm{rad} / \mathrm{s}$ & $T, \mathbf{K}$ \\
\hline \multirow{2}{*}{ Upper bound } & Upper limit & 2.02 & 1349 & 1355 \\
& Lower limit & 0.002 & 1285 & 1291 \\
\hline \multirow{2}{*}{ Lower bound } & Upper limit & - & 1051 & 1056 \\
& Lower limit & - & 736 & 739 \\
\hline
\end{tabular}

$$
\begin{aligned}
& \text { Find } \mathbf{x}=\left(x_{1}, x_{2}\right)^{T}=(w, T)^{T}, \\
& \min \quad f(\mathbf{x}), \\
& \text { subject to }\left\{\begin{array}{l}
R \geq\left[R_{0}\right]=[0.98], \\
\underline{w} \leq w \leq \bar{w}, \\
\underline{T} \leq T \leq T, \\
f(\delta) \leq[\bar{\delta}],
\end{array}\right.
\end{aligned}
$$

In MPGA algorithm, the coefficients of the optimal front-end individual, population size, evolutional generation, stop algebra, and deviation of fitness function are 0.3 , $100,200,200$, and 0.01 , respectively. Through the solution of the reliability optimization model in equation (6), the distributions of turbine blade deformation are drawn in Figure 4, and the results are listed in Table 3 . It should be noted that we conducted 10000 simulations in each probabilistic analysis involved in the turbine blade deformation reliability optimization with the developed ISRM approach.

As demonstrated in Figure 4 and Table 3, when the allowable deformation of turbine blade meet the distribution features, relative to before optimization, the means of turbine blade deformation are reduced by $0.077 \mathrm{~mm}$, and the standard deviations (StDev) of deformation are decreased by $\sim 0.0026 \mathrm{~mm}$ after optimization. The optimal values of rotational speed and temperature are $1198.62 \mathrm{rad} / \mathrm{s}$ and $1112.93 \mathrm{~K}$ as shown in Table 3. Therefore, it is demonstrated that the developed reliability optimization model, that is, the ISRM method, is effective for the reliability optimization of turbine blade deformation failure.

3.2. Method Validation. To verify the proposed ISRM method in simulation accuracy and efficiency, the overall reliability optimization of the blade is also done with MC simulation and traditional SVM method [10], based on the same parameters and computation environment. Through the comparison of methods, Tables 4 and 5 recorded the reliability degrees and optimized objective functions, respectively, in light of different number of simulations. In Table 4, the reliability degree is defined by the ratio of the number of simulations in the safety domain to the total number of simulations (1000 simulations).

From Table 4 obtained from the probabilistic failure analyses of the disk, we can acquire the following results. (i) The MC method cannot carry out the calculation which is larger than 10000 simulations, due to too-large computational burden for the FE-based probabilistic analysis, so that the MC method does not have computing time under the simulations. Thus, it is unworkable for the MC method to analyze complex structure with large-scale simulations; (ii) 


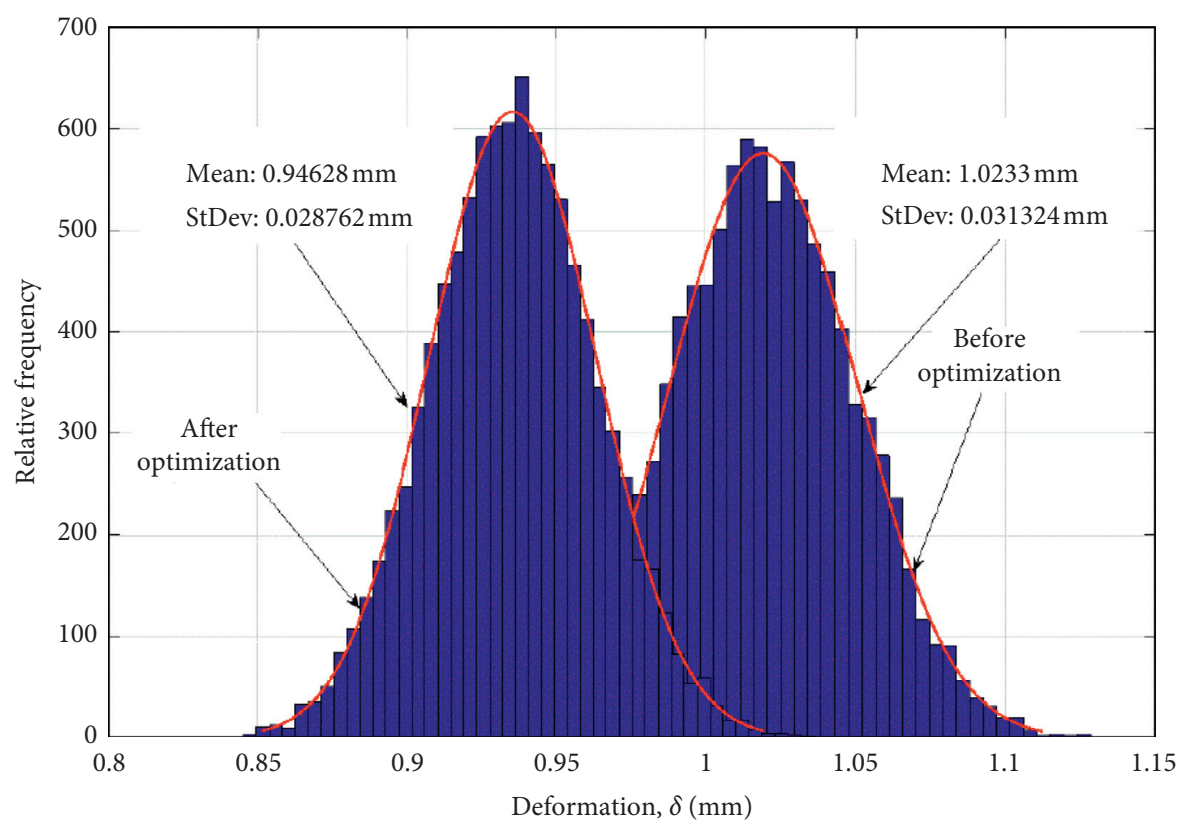

Figure 4: Distributions of blade deformation before and after optimization.

TABLE 3: Optimization results of overall blade failure.

\begin{tabular}{lcc}
\hline Design variables & Original data & Optimal values \\
\hline$\omega, \mathrm{rad} / \mathrm{s}$ & 1168 & 1198.62 \\
$T, \mathbf{K}$ & 1173.2 & 1112.93 \\
\hline
\end{tabular}

TABLE 4: Computing time and reliability degrees of blade probabilistic analysis with different methods.

\begin{tabular}{lcccccc}
\hline \multirow{2}{*}{ Number of samples } & \multicolumn{3}{c}{ Computing time (s) } & \multicolumn{3}{c}{ Reliability degree (\%) } \\
& MC method & SVM & ISRM method & MC method & SVM & ISRM method \\
\hline 100 & 54859 & 0.0111 & 0.005 & 99 & 97 & 98 \\
1000 & 340094 & 0.335 & 0.151 & 99.5 & 98.5 & 99.3 \\
10000 & - & 0.775 & 0.412 & 99.34 & 98.71 & 99.28 \\
100000 & - & 2.103 & 1.241 & - & 98.817 & 99.779 \\
\hline
\end{tabular}

TABLE 5: Optimization results of turbine blade deformation with different methods.

\begin{tabular}{|c|c|c|c|c|c|c|c|}
\hline \multirow{2}{*}{ Objective function } & \multirow{2}{*}{ Before optimization } & \multicolumn{2}{|c|}{ MC method } & \multicolumn{2}{|l|}{ SVM } & \multicolumn{2}{|c|}{ ISRM method } \\
\hline & & After optimization & Reduction & After optimization & Reduction & After optimization & Reduction \\
\hline$\delta, \mathrm{mm}$ & 1.0233 & 0.98798 & 0.03532 & 1.0023 & 0.021 & 0.93001 & 0.09329 \\
\hline$R$ & 95.53 & 96.87 & - & 97.82 & - & 98.86 & - \\
\hline
\end{tabular}

the time consumption of blade probabilistic analysis rises with the increasing MC simulations for the three methods; (iii) the time cost of the ISRM method is far less than the MC method and SVM method for the same number of simulations. Furthermore, the strength of the ISRM method in computational efficiency is more obvious with the increase of simulations. Thus, it is illustrated that the calculation efficiency of the ISRM method is far higher than the MC method and SVM method, so the developed approach is efficient in the probabilistic analysis of complex structures, instead of FE and SVM models. (iv) Under the same simulations, the reliability degrees with the ISRM method almost agree with these with the MC method. Moreover, the rise of simulations can enlarge turbine blade deformation reliability degree. It is explained that increasing the number of MC simulations can improve the precision of reliability degree, for structural probabilistic design.

As revealed in Table 5, the deformation of turbine blade is reduced by $0.09329 \mathrm{~mm}$, respectively, after the optimizations with the ISRM method. Correspondingly, the MC method lessens turbine blade deformation by $0.03532 \mathrm{~mm}$, and the SVM method declines to $0.021 \mathrm{~mm}$. The reliability index of the blade was improved from $95.53 \%$ to $96.87 \%$, $97.82 \%$, and $98.86 \%$ for the MC method, SVM method, and 
ISRM method. Obviously, the ISRM method is more effective in the reliability optimization of complex structures like turbine blade deformation.

In short, the developed ISRM method has high modeling precision and high simulation efficiency for the reliability optimization of mechanical structures. In addition, with the assist of the proposed IRSM method, we can acquire more satisfactory design results for the reliability optimization of complex structures. Therefore, it is supported that machine learning methods are promising to offer satisfying accuracy and efficiency in modeling and reliability design of structural systems.

\section{Conclusions}

The purpose of this paper is to propose a precise and efficient machine learning method, that is, ISRM method, for the reliability optimization design of complex structure. We discuss the theory and modeling of the ISRM method and the procedure of structural probabilistic optimization. In line with the ISRM method, the reliability optimization of a turbine blade is performed by considering design objective (i.e., turbine blade deformation), design variables (including material parameters and load parameters), and constraint boundary of design objective functions. The main conclusions are summarized as follows:

(1) As revealed in the reliability optimization of a turbine blade deformation failure using the ISRM method, the turbine blade deformation under operation reduced by $0.09329 \mathrm{~mm}$, which improves the reliability of turbine blade.

(2) It is illustrated in the failure probabilistic analysis of bladed disk that not only does the developed ISRM method cost limited time and high efficiency relative to the MC method and SVM method, but also its precision is acceptable in reliability degree as its optimization results are almost the same as that of the FE method with MC simulations. The high modeling precision and simulation efficiency of the ISRM method are more remarkable with the increase of simulations.

(3) The developed ISRM method is more workable in the reliability optimization of turbine blade deformation than the MC method and SVM method because the optimal design parameters and design object are preferable for larger reductions and reliability degree.

This investigation attempts to use a novel machine learning approach (i.e., ISRM method) for the reliability optimization of mechanical structures with highly precise modeling and highly efficient simulation and enrich mechanical reliability design theory.

\section{Data Availability}

The data used to support the findings of this study are available from the corresponding author upon request.

\section{Conflicts of Interest}

The authors declare that there are no conflicts of interest regarding the publication of this article.

\section{Acknowledgments}

This research was funded by the National Natural Science Foundation of China (Grant no. 51975124), Special Science and Technology Program of Civil Aircraft of China (Grant no. MJZ-Y-2018-92), and Start-Up Research Funding of Fudan University (Grant no. FDU38341).

\section{References}

[1] C. Lu, Y.-W. Feng, C.-W. Fei, and S.-Q. Bu, "Improved decomposed-coordinated Kriging modeling strategy for dynamic probabilistic analysis of multi-component structures," IEEE Transactions on Reliability, 2019.

[2] C.-W. Fei, C. Lu, and R. P. Liem, "Decomposed-coordinated surrogate modeling strategy for compound function approximation in a turbine-blisk reliability evaluation," Aerospace Science and Technology, vol. 95, p. 105466, 2019.

[3] C. Lu, Y.-W. Feng, and C.-W. Fei, "Weighted regressionbased extremum response surface method for structural dynamic fuzzy reliability analysis," Energies, vol. 12, no. 9, p. 1588, 2019.

[4] B. Keshtegar and S.-P. Zhu, "Three-term conjugate approach for structural reliability analysis," Applied Mathematical Modelling, vol. 76, pp. 428-442, 2019.

[5] C. Lu, Y.-W. Feng, R. P. Liem, and C.-W. Fei, "Improved kriging with extremum response surface method for structural dynamic reliability and sensitivity analyses," Aerospace Science and Technology, vol. 76, pp. 164-175, 2018.

[6] Z. Meng and B. Keshtegar, "Adaptive conjugate single-loop method for efficient reliability-based design and topology optimization," Computer Methods in Applied Mechanics and Engineering, vol. 344, pp. 95-119, 2019.

[7] L.-K. Song, G.-C. Bai, C.-W. Fei, and J. Wen, "Probabilistic LCF life assessment for turbine discs with DC strategy-based wavelet neural network regression," International Journal of Fatigue, vol. 119, pp. 204-219, 2019.

[8] D.-B. Meng, Y. Li, H. Huang, Z. Wang, and Y. Liu, "Reliability-based multidisciplinary design optimization using subset simulation analysis and its application in the hydraulic transmission mechanism design," Journal of Mechanical Design, vol. 137, no. 5, p. 051402, 2015.

[9] R. Liu, P. Chen, X. Zhang, and S. Zhu, "Non-shock ignition probability of octahydro-1,3,5,7-tetranitro-tetrazocine-based polymer bonded explosives based on microcrack stochastic distribution," Propellants, Explosives, Pyrotechnics, vol. 45, pp. 1-14, 2020

[10] C.-W. Fei, W.-Z. Tang, and G.-C. Bai, "Novel method and model for dynamic reliability optimal design of turbine blade deformation," Aerospace Science and Technology, vol. 39, pp. 588-595, 2014.

[11] C.-W. Fei, Y.-S. Choy, D.-Y. Hu, G.-C. Bai, and W.-Z. Tang, "Dynamic probabilistic design approach of high-pressure turbine blade-tip radial running clearance," Nonlinear Dynamics, vol. 86, no. 1, pp. 205-223, 2016.

[12] C.-Y. Zhang, Z.-S. Yuan, Z. Wang, C.-W. Fei, and C. Lu, "Probabilistic fatigue/creep optimization of turbine bladed 
disk with fuzzy multi-extremum response surface method," Materials, vol. 12, no. 12, p. 3367, 2019.

[13] L.-K. Song, C.-W. Fei, J. Wen, and G.-C. Bai, "Multi-objective reliability-based design optimization approach of complex structure with multi-failure modes," Aerospace Science and Technology, vol. 64, pp. 52-62, 2017.

[14] C. Y. Song, J. Lee, and J. Mo Choung, "Reliability-based design optimization of an FPSO riser support using moving least squares response surface meta-models," Ocean Engineering, vol. 38, no. 2-3, pp. 304-318, 2011.

[15] B. D. Youn and K. K. Choi, "A new response surface methodology for reliability-based design optimization," Computers \& Structures, vol. 82, no. 2-3, pp. 241-256, 2004.

[16] D. Meng, S. Yang, Y. Zhang, and S. P. Zhu, "Structural reliability analysis and uncertainties-based collaborative design and optimization of turbine blades using surrogate model," Fatigue \& Fracture of Engineering Materials \& Structures, vol. 42, no. 6, pp. 1219-1227, 2019.

[17] B. Keshtegar and O. Kisi, "RM5Tree: radial basis M5 model tree for accurate structural reliability analysis," Reliability Engineering \& System Safety, vol. 180, pp. 49-61, 2018.

[18] W. Song, A. Keane, J. Rees, A. Bhaskar, and S. Bagnall, "Turbine blade fir-tree root design optimisation using intelligent CAD and finite element analysis," Computers \& Structures, vol. 80, no. 24, pp. 1853-1867, 2002.

[19] W. Song and A. J. Keane, "An efficient evolutionary optimisation framework applied to turbine blade firtree root local profiles," Structural and Multidisciplinary Optimization, vol. 29, no. 5, pp. 382-390, 2005.

[20] K. D. Brabanker, J. D. Brabanter, J. A. Suykens, and B. D. Moor, "Approximate confidence and prediction intervals for least squares support vector regression," IEEE Transactions on Neural Networks, vol. 22, pp. 110-120, 2011.

[21] H. Song, K. K. Choi, I. Lee, L. Zhao, and D. Lamb, “Adaptive virtual support vector machine for reliability analysis of highdimensional problems," Structural and Multidisciplinary Optimization, vol. 47, no. 4, pp. 479-491, 2013.

[22] Z. Meng, H. Zhou, G. Li, and H. Hu, "A hybrid sequential approximate programming method for second-order reliability-based design optimization approach," Acta Mechanica, vol. 228, no. 5, pp. 1965-1978, 2017.

[23] P. Zhu, F. Pan, W. Chen, and S. Zhang, "Use of support vector regression in structural optimization: application to vehicle crashworthiness design," Mathematics and Computers in Simulation, vol. 86, pp. 21-31, 2012.

[24] Y. L. Liu, W. L. Chen, L. P. Ding, and X. P. Wang, "Response surface methodology based on support vector regression for polygon blank shape optimization design," The International Journal of Advanced Manufacturing Technology, vol. 66, pp. 1397-1405, 2013.

[25] J. Zhang, K. Wang, W. Zhu, and P. Zhong, "Least squares fuzzy one-class support vector machine for imbalanced data," International Journal of Signal Processing, Image Processing and Pattern Recognition, vol. 8, no. 8, pp. 299-308, 2015.

[26] J. Luo, Q. Liu, Y. Yang, X. Li, M.-r. Chen, and W. Cao, “An artificial bee colony algorithm for multi-objective optimisation," Applied Soft Computing, vol. 50, pp. 235-251, 2017.

[27] C.-Y. Zhang, Z. Wang, C.-W. Fei, Z.-S. Yuan, J.-S. Wei, and W.-Z. Tang, "Fuzzy multi-SVR learning model for reliabilitybased design optimization of turbine blades," Materials, vol. 12 , no. 15 , p. 2341, 2019.

[28] C. W. Fei and G. C. Bai, "Nonlinear dynamic probabilistic analysis for turbine casing radial deformation based on extremum response surface method-based support vector machine," Journal of Computational and Nonlinear Dynamics, vol. 8, p. 041004, 2013.

[29] C.-W. Fei and G.-C. Bai, "Wavelet correlation feature scale entropy and fuzzy support vector machine approach for aeroengine whole-body vibration fault diagnosis," Shock and Vibration, vol. 20, no. 2, pp. 341-349, 2013.

[30] C. Lu, Y.-W. Feng, C.-W. Fei, and S. Bu, "Decomposed-coordinated framework with enhanced extremum kriging for multicomponent dynamic probabilistic failure analyses," IEEE Access, vol. 7, pp. 163287-163300, 2019.

[31] P. Mansouri, B. Asady, and N. Gupta, "The bisection-artificial bee colony algorithm to solve fixed point problems," Applied Soft Computing, vol. 26, no. 1, pp. 143-148, 2015.

[32] N. Imanian, M. E. Shiri, and P. Moradi, "Velocity based artificial bee colony algorithm for high dimensional continuous optimization problems," Engineering Applications of Artificial Intelligence, vol. 36, no. 11, pp. 148-163, 2014.

[33] S. K. Mustafa, H. Huseyin, G. Mesut, and U. Harun, "Artificial bee colony algorithm with variable search strategy for continuous optimization," Information Sciences, vol. 300, pp. 140-157, 2015.

[34] P. Zhao, H. L. Cui, D. Woolard, K. L. Jensen, and F. A. Buot, "Simulation of resonant tunneling structures: origin of the I-V hysteresis and plateau-like structure," Journal of Applied Physics, vol. 87, no. 3, pp. 1337-1349, 2000.

[35] J. C. Helton and F. J. Davis, "Latin hypercube sampling and the propagation of uncertainty in analyses of complex systems," Reliability Engineering \& System Safety, vol. 81, no. 1, pp. 23-69, 2003.

[36] R. G. Zhao, Q. B. Li, Y. Z. Jiang et al., "Research on transition from short to long fatigue crack propagation of GH4133B superalloy used in turbine disk of aero-engine," Key Engineering Materials, vol. 697, pp. 664-669, 2016. 